\begin{tabular}{|c|l|}
\hline Title & $\begin{array}{l}\text { Establishment of a neutralization test involving reporter gene expressing virus like particles of tick-borne encephal itis } \\
\text { virus }\end{array}$ \\
\hline Author(s) & $\begin{array}{l}\text { Yoshii, Kentaro; Ikawa, Aya; Chiba, Y umiko; Omori, Y uki; Maeda, Junko; Murata, Ryo; Kariwa, Hiroaki; Takashima, } \\
\text { Ikuo }\end{array}$ \\
\hline Citation & $\begin{array}{l}\text { Journal of Virological Methods, 161(1), 173-176 } \\
\text { https:/doi.org/10.1016/.jviromet.2009.05.016 }\end{array}$ \\
\hline Issue Date & $2009-10$ \\
\hline Doc URL & http://hdl.handle.net/2115/39146 \\
\hline Type & article (author version) \\
\hline File Information & JVM161-1_p173-176.pdf \\
\hline
\end{tabular}

Instructions for use 


\section{Establishment of a neutralization test involving reporter gene-expressing virus-like particles of tick-borne encephalitis virus}

Kentaro Yoshii, Ayae Ikawa, Yumiko Chiba, Yuki Omori, Junko Maeda, Ryo Murata, Hiroaki Kariwa, Ikuo Takashima

Laboratory of Public Health, Graduate School of Veterinary Medicine, Hokkaido University, Sapporo, Hokkaido 060-0818, Japan

Corresponding author: Dr. Kentaro Yoshii

Postal address: Laboratory of Public Health, Graduate School of Veterinary Medicine, Hokkaido University, kita-18 nishi-9, kita-ku, Sapporo, Hokkaido 060-0818, Japan Tel/fax: +81-11-706-5213

E-mail: kyoshii@vetmed.hokudai.ac.jp 


\section{Summary}

Previously, a system for packaging tick-borne encephalitis virus (TBEV) subgenomic replicon RNAs into single-round infectious virus-like particles (VLPs) was developed. In the present study, VLPs were applied to measuring the levels of neutralizing antibodies against TBEV as an alternative to performing neutralization tests with live virus. As markers of VLP infection, the genes for GFP and luciferase were inserted into the TBEV replicon, which was then packaged into VLPs. The reporter genes were expressed in cells that were infected with the VLPs, and this infection was inhibited by neutralizing antibodies to TBEV. Serum samples from wild rodents were used to evaluate the neutralization test using VLPs. All the sera that were positive in the conventional neutralization test were also found to be positive in the neutralization test using VLPs, and there were highly significant correlations between the neutralization titres obtained using the native virus and those using VLPs. These results indicate that VLPs that express reporter genes represent a useful and safe alternative to conventional neutralization testing using live virus.

Keywords: tick-borne encephalitis, virus-like particles, and neutralization test 


\section{Text}

Tick-borne encephalitis virus (TBEV), which belongs to the family Flaviviridae genus Flavivirus, causes fatal encephalitis in humans with serious sequelae (Dumpis et al., 1999). TBE occurs widely across Europe, Russia, and Far-Eastern Asia, including Japan (Blaskovic et al., 1967; Korenberg and Kovalevskii, 1999; Lindgren and Gustafson, 2001; Ormaasen et al., 2001; Roggendorf et al., 1981), and more than 10,000 cases of the disease are reported annually. TBEV can be divided into three subtypes: 1) the Far-Eastern subtype, known as Russian spring summer encephalitis (RSSE) virus; 2) the European subtype, known as Central European encephalitis (CEE) virus; and 3) the Siberian subtype (Bakhvalova et al., 2000; Ecker et al., 1999). TBEV is transmitted by tick bites and is maintained in the zoonotic transmission cycle between ticks and wild vertebrate hosts, with humans acting as accidental hosts. The major tick vector of the European subtype is Ixodes ricinus, whereas $I$. persulcatus is the major tick vector for the other two viral subtypes (Ecker et al., 1999; Gaunt et al., 2001; Hayasaka et al., 2001; Lundkvist et al., 2001).

Effective diagnosis of TBE infection relies on the detection of specific antibodies. However, the presence of cross-reactive antigenic structures among flaviviruses makes it difficult to differentiate between TBEV and other flavivirus infections/vaccinations using IgG-ELISA and the HI test (Holzmann et al., 1996). For cases that involve contact with other flaviviruses or vaccinations, a neutralizing test, which is the most specific serological test, is required to confirm the diagnosis of TBE infection. However, since TBEV is classified as a biosafety level 3 or 4 virus, a high-level biocontainment facility is required to handle the live virus required for neutralization testing.

Flavivirus virions are 40-50 nm in diameter, spherical in shape, and contain a nucleocapsid and an envelope. The flavivirus genome consists of a positive-polarity, single-stranded RNA of approximately $11 \mathrm{~kb}$, which encodes three structural proteins, the core (C), premembrane (prM), and envelope (E) proteins, and seven non-structural (NS) proteins (NS1, NS2A, NS2B, NS3, NS4A, NS4B, and NS5), within a single long open reading frame that is co-translated and cleaved 
post-translationally (Chambers et al., 1990). The 5'- and 3'-untranslated regions (UTRs) have predicted secondary stem-loop structures that are implicated as cis-acting elements in viral replication, translation, and genome packaging (Gritsun et al., 1997; Proutski et al., 1997; Rauscher et al., 1997).

Subgenomic replicons have been constructed by deleting the genes for the viral structural proteins from several flaviviruses, including TBEV (Gehrke et al., 2003; Hayasaka et al., 2004; Khromykh and Westaway, 1997; Molenkamp et al., 2003; Pang et al., 2001; Scholle et al., 2004; Shi et al., 2002). Virus-like particles (VLPs) of flavivirus are produced by the complementation of replicon RNA with the viral structural genes expressed in trans (Gehrke et al., 2003; Khromykh et al., 1998; Scholle et al., 2004; Yoshii et al., 2008). The VLPs of flaviviruses are similar to the native virus with respect to their antigenic and functional characteristics. However, progeny viruses cannot be produced from VLP-infected cells due to the lack of viral structural genes. This limitation of only a single round of infectivity enables the safe handling of VLPs under BSL-2 conditions. Therefore, VLPs can be used for serological diagnosis in place of the conventional neutralization test, which uses infectious virus. In previous studies, flavivirus VLPs were used to detect virus entry, as well as the inhibition thereof by neutralizing antibody (Ansarah-Sobrinho et al., 2008; Pierson et al., 2006). In the present study, we describe a novel approach to measuring neutralizing antibodies against TBEV using VLPs that comprise encapsidated TBEV replicons that are capable of expressing reporter genes as markers of VLP infection.

Reporter genes inserted into replicons can be used as fast and easy alternatives for the assessment of genome replication. To construct TBE replicons that express reporter genes, the gene for GFP and the gene for firefly (Photinus pyralis) luciferase (luc2) were cloned separately into the deleted structural genes of the Oshima-REP plasmid, which is a TBEV replicon that was constructed from the Far-Eastern subtype TBEV strain Oshima 5-10 (Hayasaka et al., 2004), and the resulting constructs were designated as Oshima-REP-GFP2A and Oshima-REP-luc2A, respectively (Fig. 1A). The GFP and luc2 genes were amplified from pIRES2-EGFP (Clontech) and pGL4.1 (Promega), respectively. 
These constructs contain the sequence for the first 38 codons of $\mathrm{C}$, fused in-frame to the initiation codon of GFP or luc2. To ensure appropriate processing of the reporter gene from the signal sequence of NS1, the foot and mouse disease virus (FMDV) 2A coding sequence (2A) was inserted between the C-terminus of the reporter gene and the NS1 signal sequence. The 2A protein is a 17-aa autoproteolytic peptide that has been shown to mediate the efficient cleavage of C-terminally fused heterologous protein sequences (Percy et al., 1994).

Oshima-REP-GFP2A and Oshima-REP-luc2A were prepared for run-off transcription by digestion with SpeI, and the resulting template DNAs were transcribed in vitro using the mMESSAGE mMACHINE SP6 Kit (Ambion), as described previously (Hayasaka et al., 2004). The synthesised RNA was transfected into BHK cells using the TransIT-mRNA transfection kit (Mirus Bio).

GFP was detected in the BHK cells by fluorescence microscopy as early as $24 \mathrm{~h}$ after transfection with the Oshima-REP-GFP2A replicon RNA (Fig. 1B). The GFP expression persisted at $96 \mathrm{~h}$ post-transfection. To test the Oshima-REP-luc2A replicon, BHK cells were transfected with the in vitro-transcribed RNA and the kinetics of replicon RNA replication and luciferase activity were analysed (Fig. 1C). Luciferase activity was detected using the Luciferase Assay System (Promega), and the replicon RNAs were quantified by Real-Time RT-PCR using primers and the TaqMan probe, as described previously (Schwaiger and Cassinotti, 2003). The levels of luciferase activity in the transfected cells increased significantly as the levels of replicon RNA increased (Fig. 1C).

The pcTBECME plasmid, which contains the TBEV virus C-prM-E genes (Yoshii et al., 2005), was used for the expression of TBEV structural proteins to be used for packaging the replicon RNA. To prepare the VLPs, $293 \mathrm{~T}$ cells were first transfected with Oshima-REP-GFP2A or Oshima-REP-luc2A, followed 8 h later by a second transfection with the pcTBECME plasmid, as described previously (Yoshii et al., 2005). Following the second transfection, the culture supernatant that contained the VLPs was harvested and used to infect naïve cells. At 48 h post-infection, GFP fluorescence was observed for the cells that were infected with the VLPs that contained 
Oshima-REP-GFP2A (GFP-VLPs) (Fig. 2A, upper panel: no antibody). As shown in Fig. 2B, sequential transfection with the Oshima-REP-luc2A replicon and the pcTBECME plasmid resulted in the production of VLPs, and luciferase expression was confirmed in the cells that were infected with VLPs that contained the Oshima-REP-luc2A replicon (luc-VLPs).

In previous studies, the envelope glycoproteins of the VLPs exhibited the same antigenicities as those of the authentic virion (Yoshii et al., 2005; Yoshii et al., 2008). To apply the VLPs that express reporter genes to neutralization testing, we examined whether infection with VLPs was neutralized by virus-specific neutralizing antibodies. Prior to infection, the GFP-VLPs or the luc-VLPs were incubated with the anti-TBE E protein monoclonal antibody $1 \mathrm{H} 4$, which has neutralizing activity against TBEV (Komoro et al., 2000). As shown in Fig. 2A, the number of GFP-positive cells was reduced by incubation of the GFP-VLPs with 1H4, whereas no reduction was observed after incubation of the GFP-VLPs with control normal mouse serum. Similar results were obtained for luc-VLPs incubated with 1H4 (Fig. 2C). An antibody-dependent reduction in luciferase activity was also observed in the VLP-infected cells.

To evaluate whether the VLPs that express reporter genes can be used in neutralization testing as a substitute for native virions, serum samples with neutralizing antibodies derived from 28 wild rodents, which were collected in a TBE-endemic area of Hokkaido, Japan, were tested. The results for the neutralization tests using VLPs that express reporter genes were compared with those for neutralization tests using the native virus. Prior to the infection of BHK cells, the VLPs were incubated with serum samples that were diluted serially. The neutralizing antibody titre was determined as the reciprocal of the highest serum dilution that reduced the number of viral foci by $50 \%$ or more when using the native virus, that reduced the number of GFP-positive cells by $50 \%$ or more when using the GFP-VLPs or that reduced the level of luciferase activity by $80 \%$ or more when using the luc-VLPs. The correlations between the neutralizing antibody titres obtained using native TBEV and the GFP-VLPs or luc-VLPs are shown in Fig. 3. There was a highly significant correlation between the neutralizing antibody titre obtained using the native virus and those titres obtained using 
GFP-VLPs and luc-VLPs $[\mathrm{r}=0.83277(\mathrm{P}<0.0001)$ and $\mathrm{r}=0.78834(\mathrm{P}<0.00001)$, respectively]. Fifteen serum samples that were found to be negative by the neutralization test with the VLPs were also found to be negative by the conventional neutralization test with the native virus.

The application of reporter gene-expressing VLPs to neutralization tests offers several advantages over neutralization tests using live virus. The standard method for neutralization testing of TBEV is the plaque or focus reduction neutralization test (PRNT or FRNT). The PRNT/FRNT is the most specific serological test, although this approach has several limitations. The formation of plaques or foci depends on the cell type and culture conditions, and the number of plaques or foci must be estimated manually by investigators. Thus, the results may be somewhat subjective when the plaque sizes and/or morphologies are variable. In addition, the PRNT/FRNT uses live viruses, which must be handled by skilled investigators in a high-level biocontainment facility. The neutralization test using VLPs can complement the features of the PRNT/FRNT. The reporter genes can be useful markers for quantifying cell infection by VLPs, and the neutralization test using VLPs can be performed both rapidly (in 2 days) and on a large scale using a 96-well format. Furthermore, the single-round infectivity feature of VLPs enables safe handling under BSL-2 conditions. Neutralization testing using VLPs can be also applied to serological diagnoses of other flaviviruses though the construction of pseudo-typed VLPs. In a previous study, pseudo-typed VLPs with envelope proteins derived from Japanese encephalitis virus were constructed (Yoshii et al., 2008). The antigenic characteristics of the pseudo-typed VLPs are similar to those of the native virus. By replacing the structural proteins with those from other flaviviruses, neutralization testing using VLPs can be adapted to the detection of antibodies with specificities for a variety of flaviviruses.

In summary, we show that VLPs that express reporter genes can be applied to neutralization testing for the detection of specific antibodies against TBEV. A significant correlation was observed between the neutralizing antibody titre obtained using the native virus and that using VLPs. These data demonstrate that neutralization testing using VLPs is a useful approach for the diagnosis of TBE infection and as a substitute for conventional neutralization testing with live viruses. 


\section{Acknowledgements}

We thank Dr. Akihiko Maeda of the Laboratory of Prion Diseases, Graduate School of Veterinary Medicine, Hokkaido University, for helpful discussions regarding the application of VLPs to serological diagnosis. This work was supported by Grants-in-Aid for Scientific Research from the Ministry of Education, Science, Sports, and Culture of Japan, and Health Sciences Grants for Research on Emerging and Re-emerging Infectious Disease from the Ministry of Health, Labour, and Welfare of Japan.

\section{References}

Ansarah-Sobrinho, C., Nelson, S., Jost, C.A., Whitehead, S.S.Pierson, T.C., 2008. Temperature-dependent production of pseudoinfectious dengue reporter virus particles by complementation. Virology 381, 67-74.

Bakhvalova, V.N., Rar, V.A., Tkachev, S.E., Matveev, V.A., Matveev, L.E., Karavanov, A.S., Dobrotvorsky, A.K.Morozova, O.V., 2000. Tick-borne encephalitis virus strains of Western Siberia. Virus Res 70, $1-12$.

Blaskovic, D., Pucekova, G., Kubinyi, L., Stupalova, S.Oravcova, V., 1967. An epidemiological study of tick-borne encephalitis in the Tribec region: 1953-63. Bull World Health Organ 36, Suppl 1:89-94.

Chambers, T.J., Hahn, C.S., Galler, R.Rice, C.M., 1990. Flavivirus genome organization, expression, and replication. Annu Rev Microbiol 44, 649-88.

Dumpis, U., Crook, D.Oksi, J., 1999. Tick-borne encephalitis. Clin Infect Dis 28, 882-90.

Ecker, M., Allison, S.L., Meixner, T.Heinz, F.X., 1999. Sequence analysis and genetic classification of tick-borne encephalitis viruses from Europe and Asia. J Gen Virol 80, 179-85.

Gaunt, M.W., Sall, A.A., de Lamballerie, X., Falconar, A.K., Dzhivanian, T.I.Gould, E.A., 2001.

Phylogenetic relationships of flaviviruses correlate with their epidemiology, disease association and biogeography. J Gen Virol 82, 1867-76.

Gehrke, R., Ecker, M., Aberle, S.W., Allison, S.L., Heinz, F.X.Mandl, C.W., 2003. Incorporation of tick-borne encephalitis virus replicons into virus-like particles by a packaging cell line. J Virol 77, 8924-33.

Gritsun, T.S., Venugopal, K., Zanotto, P.M., Mikhailov, M.V., Sall, A.A., Holmes, E.C., Polkinghorne, I., Frolova, T.V., Pogodina, V.V., Lashkevich, V.A.Gould, E.A., 1997. Complete sequence of two tick-borne flaviviruses isolated from Siberia and the UK: analysis and significance of the 5 ' and 
3'-UTRs. Virus Res 49, 27-39.

Hayasaka, D., Ivanov, L., Leonova, G.N., Goto, A., Yoshii, K., Mizutani, T., Kariwa, H.Takashima, I., 2001. Distribution and characterization of tick-borne encephalitis viruses from Siberia and far-eastern Asia. J Gen Virol 82, 1319-28.

Hayasaka, D., Yoshii, K., Ueki, T., Iwasaki, T.Takashima, I., 2004. Sub-genomic replicons of Tick-borne encephalitis virus. Arch Virol 149, 1245-56.

Holzmann, H., Kundi, M., Stiasny, K., Clement, J., McKenna, P., Kunz, C.Heinz, F.X., 1996. Correlation between ELISA, hemagglutination inhibition, and neutralization tests after vaccination against tick-borne encephalitis. J Med Virol 48, 102-7.

Khromykh, A.A., Varnavski, A.N.,Westaway, E.G., 1998. Encapsidation of the flavivirus kunjin replicon RNA by using a complementation system providing Kunjin virus structural proteins in trans. J Virol 72, 5967-77.

Khromykh, A.A.Westaway, E.G., 1997. Subgenomic replicons of the flavivirus Kunjin: construction and applications. J Virol 71, 1497-505.

Komoro, K., Hayasaka, D., Mizutani, T., Kariwa, H.Takashima, I., 2000. Characterization of monoclonal antibodies against Hokkaido strain tick-borne encephalitis virus. Microbiol Immunol 44, 533-6.

Korenberg, E.I.Kovalevskii, Y.V., 1999. Main features of tick-borne encephalitis eco-epidemiology in Russia. Zentralbl Bakteriol 289, 525-39.

Lindgren, E.Gustafson, R., 2001. Tick-borne encephalitis in Sweden and climate change. Lancet 358, $16-8$.

Lundkvist, K., Vene, S., Golovljova, I., Mavtchoutko, V., Forsgren, M., Kalnina, V.Plyusnin, A., 2001. Characterization of tick-borne encephalitis virus from Latvia: evidence for co-circulation of three distinct subtypes. J Med Virol 65, 730-5.

Molenkamp, R., Kooi, E.A., Lucassen, M.A., Greve, S., Thijssen, J.C., Spaan, W.J.Bredenbeek, P.J., 2003. Yellow fever virus replicons as an expression system for hepatitis $\mathrm{C}$ virus structural proteins. $\mathrm{J}$ Virol 77, 1644-8.

Ormaasen, V., Brantsaeter, A.B., Moen, E.W., 2001. [Tick-borne encephalitis in Norway]. Tidsskr Nor Laegeforen 121, 807-9.

Pang, X., Zhang, M., Dayton, A.I., 2001. Development of Dengue virus type 2 replicons capable of prolonged expression in host cells. BMC Microbiol 1, 18.

Percy, N., Barclay, W.S., Garcia-Sastre, A., Palese, P., 1994. Expression of a foreign protein by influenza A virus. J Virol 68, 4486-92.

Pierson, T.C., Sanchez, M.D., Puffer, B.A., Ahmed, A.A., Geiss, B.J., Valentine, L.E., Altamura, L.A., Diamond, M.S., Doms, R.W., 2006. A rapid and quantitative assay for measuring antibody-mediated neutralization of West Nile virus infection. Virology 346, 53-65.

Proutski, V., Gould, E.A., Holmes, E.C., 1997. Secondary structure of the 3' untranslated region of flaviviruses: similarities and differences. Nucleic Acids Res 25, 1194-202.

Rauscher, S., Flamm, C., Mandl, C.W., Heinz, F.X., Stadler, P.F., 1997. Secondary structure of the 
3'-noncoding region of flavivirus genomes: comparative analysis of base pairing probabilities. Rna 3, 779-91.

Roggendorf, M., Goldhofer, E., Heinz, F.X., Epp, C.Deinhardt, F., 1981. [Epidemiology of tick-borne encephalitis in Southern Germany (author's transl)]. MMW Munch Med Wochenschr 123, 1407-11.

Scholle, F., Girard, Y.A., Zhao, Q., Higgs, S., Mason, P.W., 2004. trans-Packaged West Nile virus-like particles: infectious properties in vitro and in infected mosquito vectors. J Virol 78, 11605-14.

Schwaiger, M.Cassinotti, P., 2003. Development of a quantitative real-time RT-PCR assay with internal control for the laboratory detection of tick borne encephalitis virus (TBEV) RNA. J Clin Virol 27, 136-45.

Shi, P.Y., Tilgner, M., Lo, M.K., 2002. Construction and characterization of subgenomic replicons of New York strain of West Nile virus. Virology 296, 219-33.

Yoshii, K., Goto, A., Kawakami, K., Kariwa, H.,Takashima, I., 2008. Construction and application of chimeric virus-like particles of tick-borne encephalitis virus and mosquito-borne Japanese encephalitis virus. J Gen Virol 89, 200-11.

Yoshii, K., Hayasaka, D., Goto, A., Kawakami, K., Kariwa, H., Takashima, I., 2005. Packaging the replicon RNA of the Far-Eastern subtype of tick-borne encephalitis virus into single-round infectious particles: development of a heterologous gene delivery system. Vaccine 23, 3946-56. 


\section{Figure legends}

Fig. 1. (A) Schematic representation of the TBEV genome and replicon constructs. TBE replicon regions were inserted under the control of the SP6 promoter. The genes for GFP and luciferase were inserted between the $\mathrm{C}$ and $\mathrm{E}$ protein-encoding regions of the TBEV genome. The foot and mouse disease virus (FMDV) 2A coding sequence (2A) was inserted between the C-terminus of the GFP or luciferase gene and the NS1 signal sequence. (B, C) Expression of reporter genes in TBEV replicons. BHK cells were transfected with Oshima-REP-GFP2A or Oshima-REP-luc2A and monitored for reporter gene expression. GFP fluorescence was observed $24 \mathrm{~h}$ post-transfection in the cells that were transfected with Oshima-REP-GFP2A (B). Luciferase activity and replicon RNA replication were monitored from $4 \mathrm{~h}$ to $36 \mathrm{~h}$ post-transfection with Oshima-REP-luc2A. The replicon RNAs were quantified by Real-Time PCR. Luciferase activity is expressed in Raw Luciferase Units (RLU).

Fig. 2. Infection with VLPs that express reporter genes. (A) BHK cells were infected with the GFP-VLPs, and GFP fluorescence was confirmed at $48 \mathrm{~h}$ post-infection (upper panel). To investigate the neutralization of VLP infection, GFP-VLPs were incubated with anti-TBE E protein monoclonal antibody $1 \mathrm{H} 4$ or normal mouse serum (lower panel) prior to infection. (B) BHK cells were infected with luc-VLPs, and luciferase activity was detected $12-72 \mathrm{~h}$ post-infection. Luciferase activity is expressed in Raw Luciferase Units (RLU). (C) Neutralization of VLP infection. BHK cells were infected with VLPs after incubation with serial dilutions of monoclonal antibody $1 \mathrm{H} 4$. The level of luciferase activity was detected $48 \mathrm{~h}$ post-infection and is expressed in Raw Light Units (RLU).

Fig. 3. Correlation between the neutralizing antibody titre obtained using native virus and those obtained using GFP-VLPs (upper panel) and luc-VLPs (lower panel). Twenty-eight serum samples collected from wild rodents in a TBEV-endemic area were used. 
The English in this document has been checked by at least two professional editors, both native speakers of English. For a certificate, see:

http://www.textcheck.com/certificate/P8NjAH 


\section{Figure 1}

A.

TBEV genome

5'UTR

3'UTR

\begin{tabular}{|l|l|l|l|l|l|l|l|l|}
\hline C prM & E & NS1 & $2 \mathrm{~A}$ & $2 \mathrm{~B}$ & 3 & $4 \mathrm{~A}$ & $4 \mathrm{~B}$ & 5 \\
\hline
\end{tabular}

Oshima-REP-GFP2A

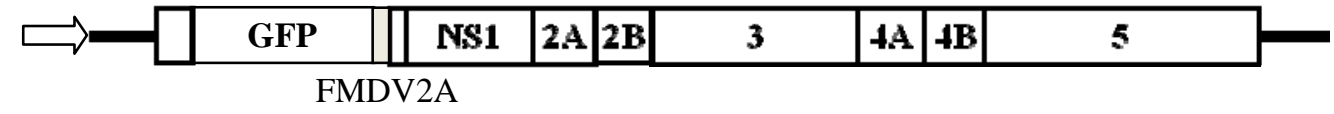

Oshima-REP-luc2A

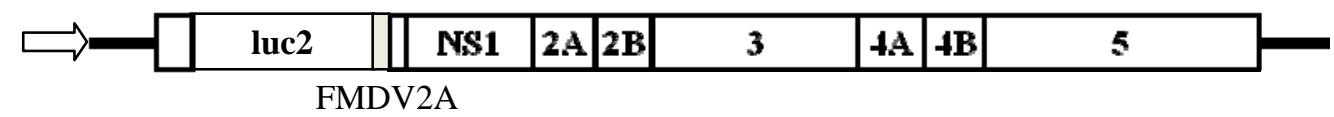

B.

Oshima-REP-GFP2A

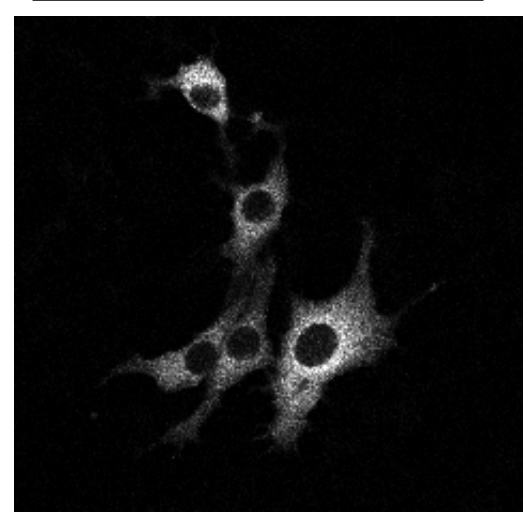

C. Oshima-REP-luc2A

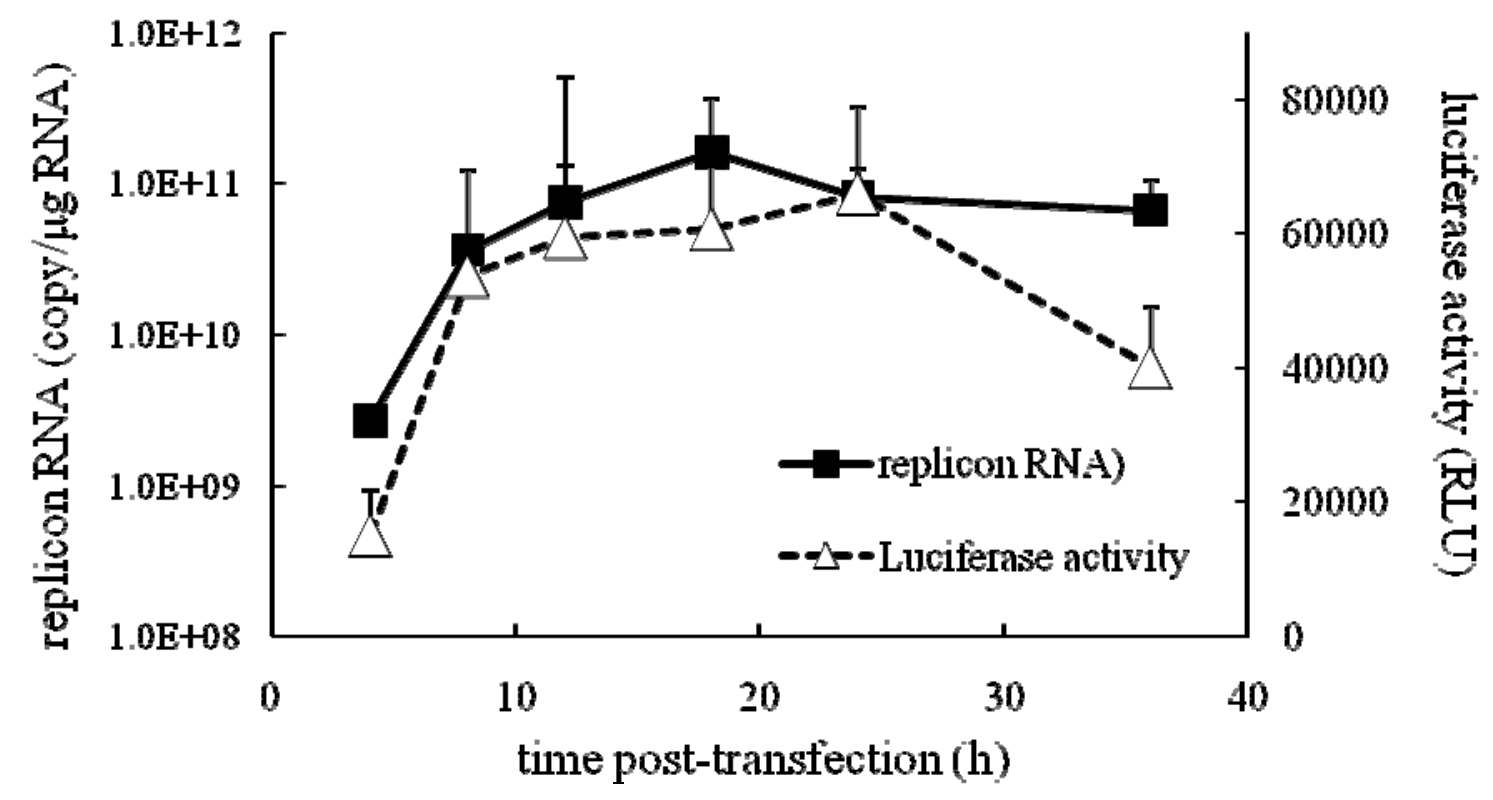


Figure 2

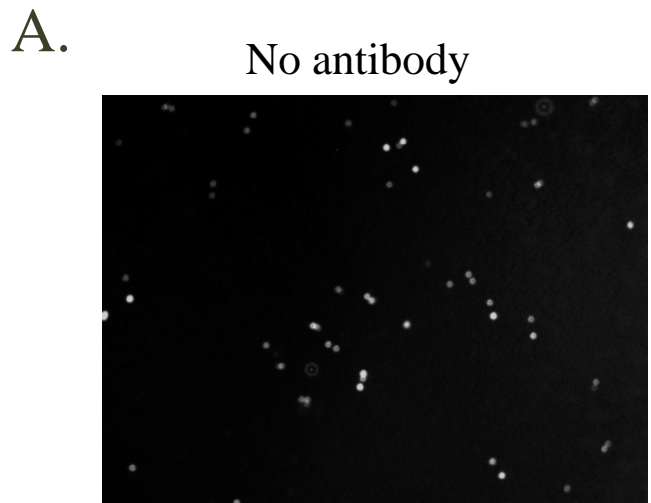

1H4

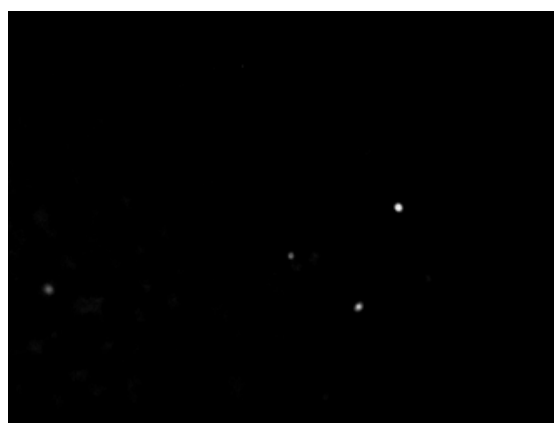

B.

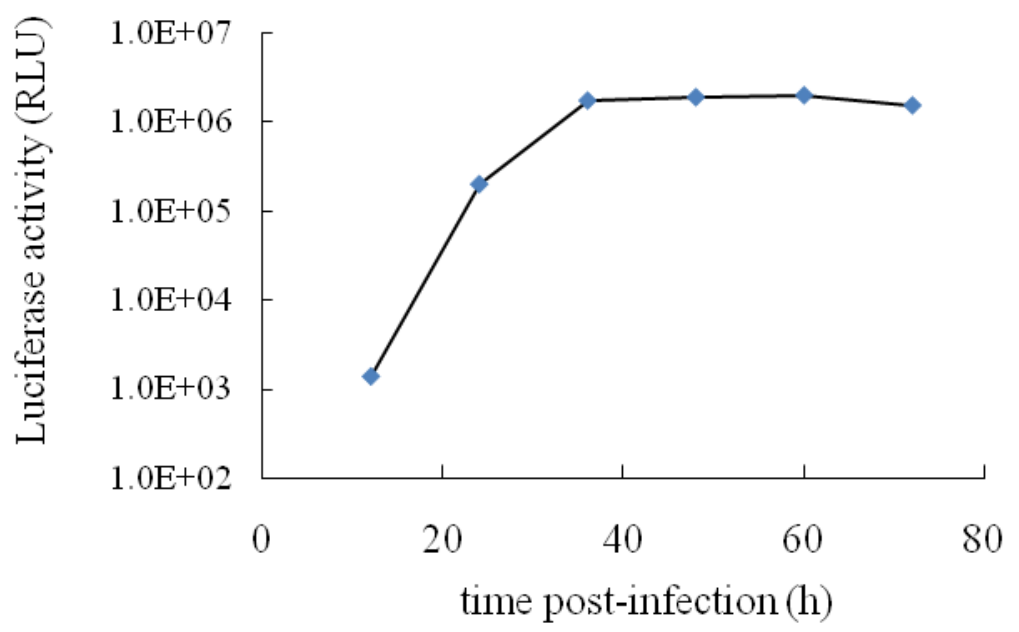

C.

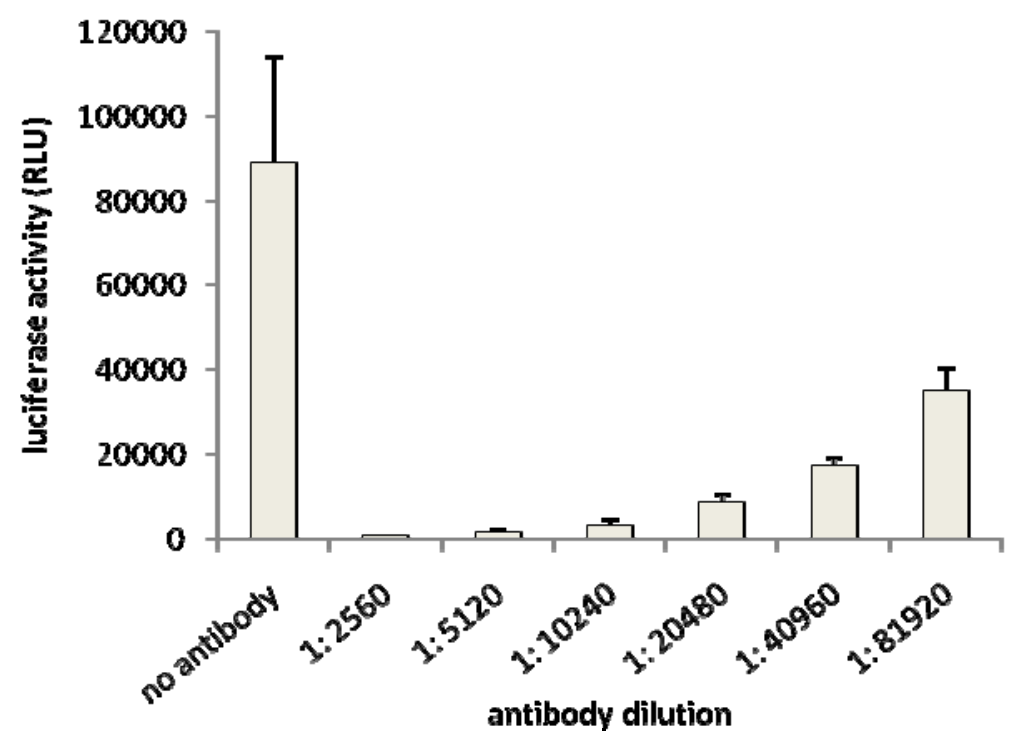


Figure 3

\section{GFP-VLPs}

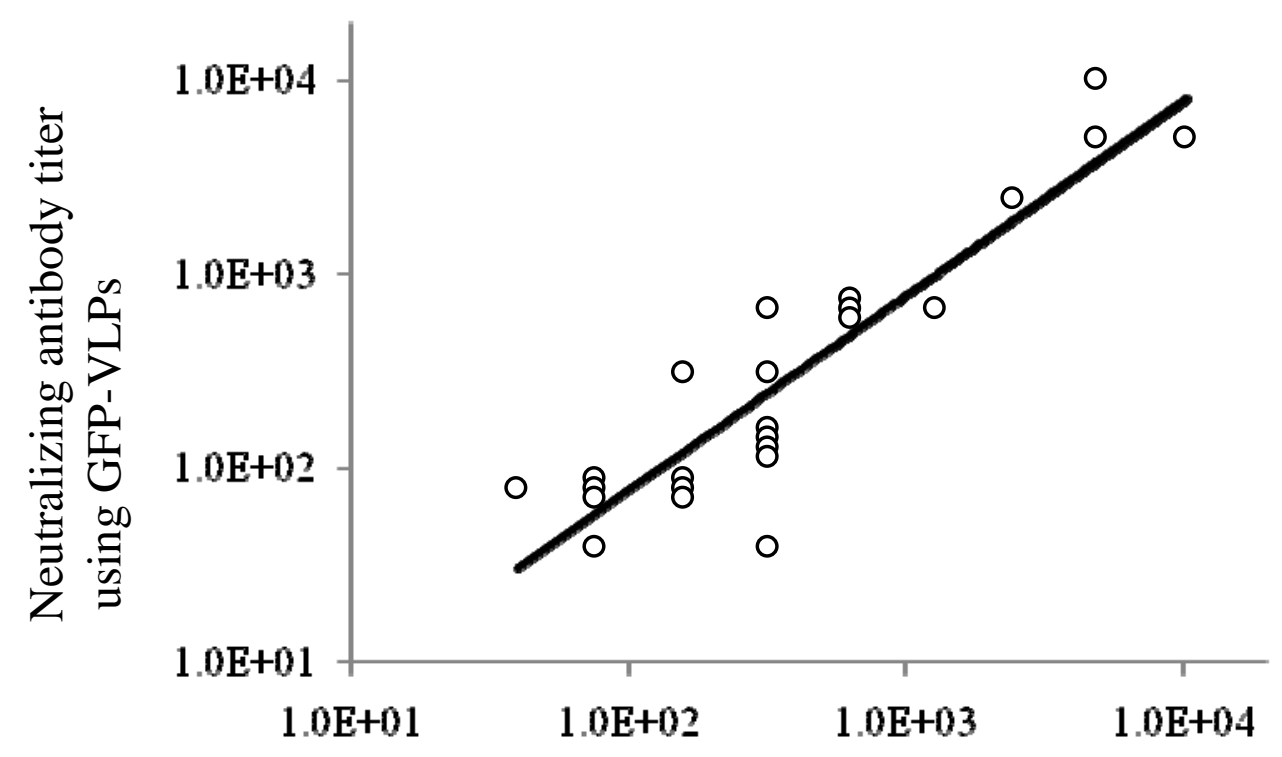

Neutralizing antibody titer using virus

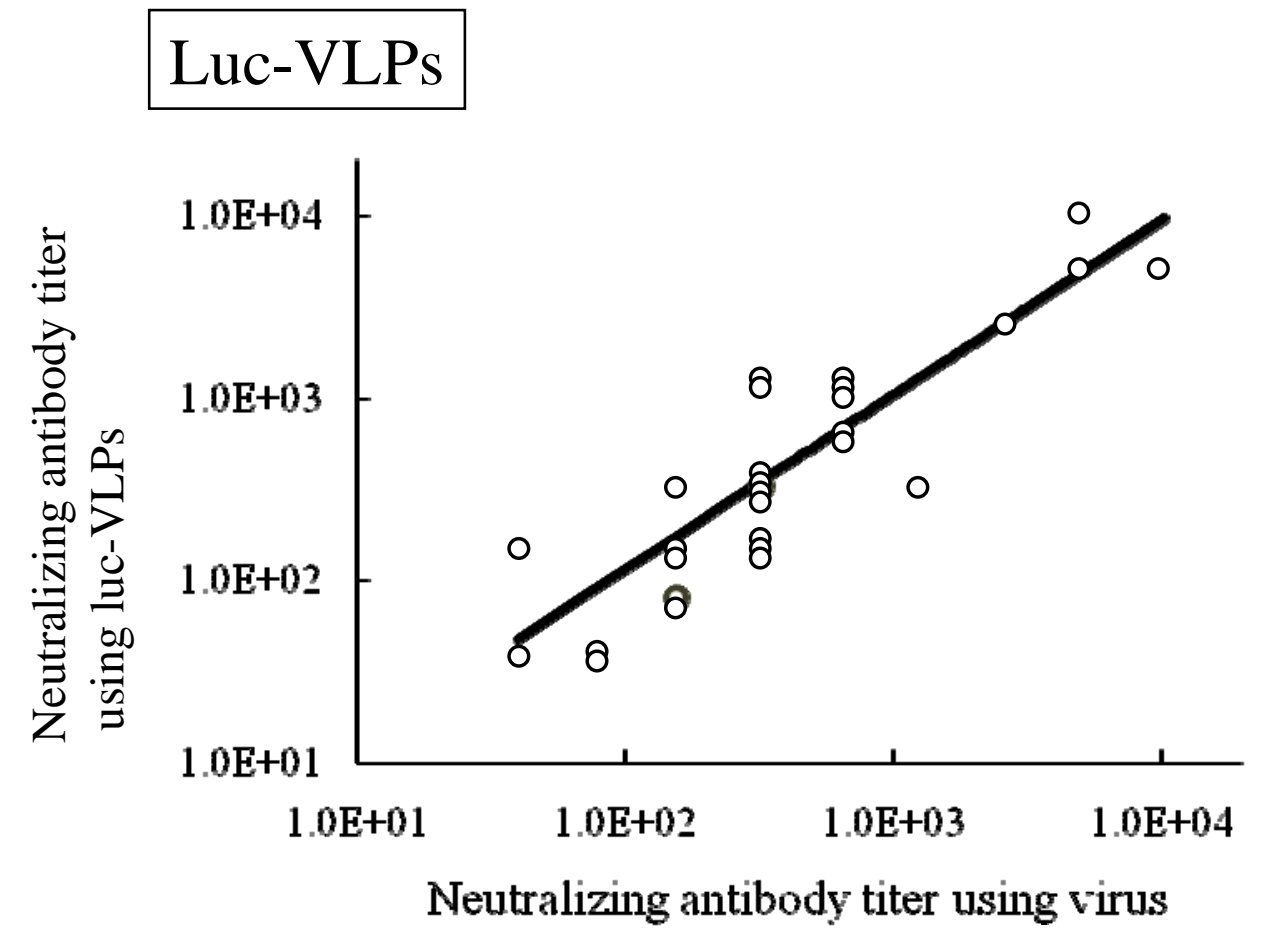

